\title{
Monensin suppresses cell proliferation and invasion in ovarian cancer by enhancing MEK1 SUMOylation
}

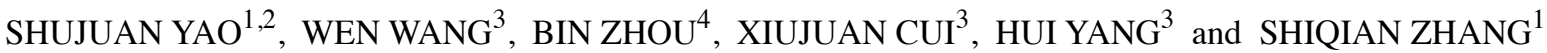 \\ ${ }^{1}$ Department of Obstetrics and Gynecology, Qilu Hospital, Cheeloo College of Medicine, Shandong University, \\ Jinan, Shandong 250012; ${ }^{2}$ Department of Obstetrics and Gynecology, Affiliated Hospital of Shandong University \\ of TCM, Jinan, Shandong 250014; ${ }^{3}$ Department of Obstetrics and Gynecology, Jining Medical University \\ Affiliated Tengzhou Central People's Hospital, Tengzhou, Shandong 277599; ${ }^{4}$ Department of Obstetrics \\ and Gynecology, Tai'an City Central Hospital, Tai'an, Shandong 271000, P.R. China
}

Received October 18, 2020; Accepted June 30, 2021

DOI: $10.3892 /$ etm.2021.10826

\begin{abstract}
Ovarian cancer is the most lethal gynecologic malignancy, and is usually diagnosed at an advanced stage. Most patients relapse within 12-24 months and die from progressive chemotherapy-resistant diseases. Significant progress has been made in developing new targeted therapies for human cancer, including ovarian cancer. However, an effective alternative to drug development is to repurpose drugs. The present study investigated the possibility of reusing the antibiotic monensin as an anti-ovarian cancer drug. After applying a series of titrated monensin on a panel of ovarian cancer cell lines, the growth, migration and invasion of cells were explored. Multiple signaling molecules associated with epithelial-to-mesenchymal transition were also regulated by monensin. The mitogen-activated protein kinase (MEK)extracellular signal-regulated kinase (ERK) pathway was further found to be the key regulator affected by monensin. Additionally, monensin enhanced the MEK1 SUMOylation in vitro and in vivo, and the SUMOylation degree depended on time and dose. Xenograft studies verified that monensin effectively inhibited xenograft tumor growth by increasing the SUMOylation of MEK1. The aforementioned results suggested that monensin is a good candidate for anti-ovarian cancer by enhancing MEK1 SUMOylation and inhibiting the MEK-ERK pathway.
\end{abstract}

Correspondence to: Professor Shiqian Zhang, Department of Obstetrics and Gynecology, Qilu Hospital, Cheeloo College of Medicine, Shandong University, 107 West Wenhua Road, Jinan, Shandong 250012, P.R. China

E-mail: zhangshiqian370112@126.com

Abbreviations: EMT, epithelial-to-mesenchymal transition; MEK, mitogen-activated protein kinase; ERK, extracellular signalregulated kinase

Key words: monensin, ovarian cancer, SUMOylation, MEK1

\section{Introduction}

Ovarian cancer is the most common lethal gynecological malignancy. More than two-thirds of patients are diagnosed at a late stage [Federation Internationale of Gynecologie and Obstetrigue (FIGO), stages III or IV], and the five-year survival rate is $<30 \%(1,2)$. Carcinoma diagnosed in situ could elevate the five-year survival rate to $92 \%(1,3)$, but a diverse group of tumors in epithelial ovarian cancer impedes the early detection and new therapeutic approaches. Accumulating evidence has indicated that epithelial-to-mesenchymal transition (EMT) plays a crucial role in the development of ovarian cancer, which is a major driver of mortality in patients with cancer, whereas EMT is upregulated by the mitogen-activated protein kinase (MEK)-extracellular signal-regulated kinase (ERK) pathway $(4,5)$. The ERK/MAPK cascade mediates mitogenic signaling and is essential for the control of cell fate, differentiation and proliferation, which is frequently hyperactivated in human cancer (6).

MEK/ERK pathways are regulated by various mechanisms $(6,7)$. Protein phosphorylation is one of the most important formats: Activated Raf phosphorylation and activates MEK1 and MEK2, which then phosphorylates ERK1 and ERK2, respectively $(8,9)$. The protein Small ubiquitin-like modifier (SUMO) is an important pathway for modulating cellular function (10). SUMOs are small ubiquitin-like modifiers containing 92-97-amino-acid polypeptides (11). Similar to phosphorylation, SUMOylation is a reversible and dynamic process involving the activation of enzyme and cysteine proteases, which in turn modulate the function, subcellular localization, complex formation and/or stability of substrate proteins.

Monensin (Rumensin ${ }^{\mathrm{TM}}$ ) is a polyether ionophore antibiotic secreted by the bacterium Streptomyces cinnamonensis $(12,13)$. Previous studies have demonstrated that monensin exerts cytotoxic effects against several types of cancer cells (14-16), but the underlying mechanism has not yet been thoroughly explored.

In the present study, the anticancer activity of monensin against human ovarian cancer cells was investigated by using a series of human ovarian cancer lines. Further studies indicated 
that monensin effectively abrogated MEK SUMOylation, resulting in the enhanced activation of the ERK pathway, whereas SUMOylation faded with medium change and monensin withdrawal. Overall, the present study results revealed a previously unknown mechanism of monensin against ovarian carcinogenesis.

\section{Materials and methods}

Cell culture. Human ovarian cancer cell lines SK-OV-3, A2780, OVCAR3 and CAOV-3 were purchased from the American Type Culture Collection. The aforementioned cells were maintained in complete Dulbecco's modified Eagle's medium (DMEM; Gibco; Thermo Fisher Scientific, Inc.) supplemented with $10 \%$ fetal bovine serum (FBS; Invitrogen; Thermo Fisher Scientific, Inc.) at $37^{\circ} \mathrm{C}$ in $5 \% \mathrm{CO}_{2}$.

Chemicals. Monensin (cat. no. S2324; Selleck Chemicals) were added to the cells following the indicated concentration. Monensin was dissolved in ethanol at $50 \mathrm{mM}$ for storage in $-80^{\circ} \mathrm{C}$. When used, monensin was diluted 1:1,000 with DMEM, and added to cellular medium based on the total volume for working solutions, such as $0.2,1$ and $5 \mu \mathrm{M}$.

Cell proliferation assay. Cell proliferation in response to monensin in vitro was assessed by Cell Counting Kit-8 (CCK-8; Dojindo Molecular Technologies, Inc.) assay according to the manufacturer's instructions. Ovarian cancer cells were cultured at a density of $5 \times 10^{3}$ per well in FBS $10 \%(\mathrm{v} / \mathrm{v})$ DMEM medium on 96 -well plate at $37^{\circ} \mathrm{C}$. After $24 \mathrm{~h}$ of incubation, the culture medium were treated with monensin for 24,48 and $72 \mathrm{~h}$ respectively. Then, $10 \mu \mathrm{l}$ CCK- 8 reagent was added to each well followed by incubation at $37^{\circ} \mathrm{C}$ for $2 \mathrm{~h}$. Absorbance was measured using a microplate reader (Molecular Devices, LLC) at $450 \mathrm{~nm}$ with a reference wavelength of $650 \mathrm{~nm}$.

The CellTiter-Glo (CTG) Luminescent Cell Viability assay is a homogenous method of determining the number of viable cells in culture based on the quantitation of the ATP present, which signals the presence of metabolically active cells. The cells were cultured in 96-well plates and treated with monensin for 24,48 and $72 \mathrm{~h}$ respectively. Before luminescence recording, $100 \mu \mathrm{l}$ CTG reagent (Promega Corporation) was added to each well, and the contents were mixed for $2 \mathrm{~min}$ on a shaker to induce cell lysis. The plate was equilibrated to room temperature for $10 \mathrm{~min}$, and the luminescence signal was recorded on an Ascent luminometer (Thermo Labsystems) according to the manufacturer's protocols. Data was recorded as the mean $\pm \mathrm{SD}$ of three independent experiments.

Cell wounding assay. Exponentially growing ovarian cancer cells were seeded onto 6-well plates until $80 \%$ confluence was reached. A sterile tip was used to create a wound in a cell monolayer, which was then washed 3 times to remove non-adherent cells. Fresh medium with $10 \%$ FBS was then added to the cultures. Images were captured at 0,24 , and $48 \mathrm{~h}$ after scratching. Photoshop software (Adobe Photoshop CS6; Adobe Systems, Inc.) was used to measure the distance of the wound at each time point, and the wound healing rate was calculated as follows: Wound healing rate $=($ control group wound width - sample group wound width)/control group wound width $\mathrm{x} 100 \%$. Data was recorded as the mean $\pm \mathrm{SD}$ of three independent experiments.

Transwell and invasion assay. Ovarian cancer cells were seeded onto the upper chamber with $80 \%$ confluence. Ovarian cancer cells were starved by incubating in serum-free medium for $24 \mathrm{~h}$. Culture inserts of $8-\mu \mathrm{m}$ pore size (Transwell, Costar; Corning, Inc.) were placed onto the wells of 24-well culture plates. The solution containing the test agent was placed below the permeable cell membrane, and $1 \times 10^{5}$ cells were seeded onto the upper chamber. After $48 \mathrm{~h}$ of incubation at $37^{\circ} \mathrm{C}$ with $5 \%$ $\mathrm{CO}_{2}$, the number of cells that had migrated through the pores were fixed with $4 \%$ paraformaldehyde at room temperature (RT) for $20 \mathrm{~min}$, and stained with $0.5 \%$ crystal violet for $3 \mathrm{~min}$ at RT. The photos were imaged at $\times 200$ magnification under an inverted microscope (NIB-100F; Ningbo Yongxin Optics Co., Ltd.), and the number of invading cells was determined with ImageJ software 1.42 (National Institutes of Health).

For the cell migration assay, culture inserts of $8-\mu \mathrm{m}$ pore size were coated with Matrigel (BD Biosciences; $100 \mu \mathrm{g} / \mathrm{well}$ ) and placed onto the wells of 24 -well culture plates. The subsequent steps were as aforementioned for the Transwell experiments. The number of migrated cells were imaged at x200 magnification under an inverted microscope (NIB-100F; Ningbo Yongxin Optics Co., Ltd.), and determined with ImageJ, and data was recorded as the mean $\pm \mathrm{SD}$ of three independent experiments.

Cell cycle analysis. Exponentially growing ovarian cancer cells were seeded onto 6 -well plates at a density of $5 \times 10^{4}$ and allowed to culture for $24 \mathrm{~h}$. The cells were later treated with various concentrations of monensin and stored for $48 \mathrm{~h}$. After treatment, cells were harvested with trypsin, pelleted by centrifugation, and washed with PBS. The cells were resuspended in PBS and fixed in ice-cold $70 \%$ ethanol for $24 \mathrm{~h}$ at $4^{\circ} \mathrm{C}$. After fixation, cells were centrifuged at $2,500 \mathrm{x} \mathrm{g}$ for $30 \mathrm{sec}$ and washed twice with PBS at RT, and then $1 \mathrm{ml}$ propidium iodide master mix solution (40 $\mu 1$ PI, $10 \mu 1$ RNase and $950 \mu \mathrm{lBS}$ ) were added and incubated at $37^{\circ} \mathrm{C}$ for $30 \mathrm{~min}$ in darkness. The cell cycle arrest was analyzed by flow cytometry (Accuri C6, BD Biosciences). The percentage of cell populations in the $\mathrm{G}_{1}, \mathrm{~S}$, and $\mathrm{G}_{2} / \mathrm{M}$ phases was analyzed with Modfit LT 4.0 software (Verity Software House, Inc.). Measurements were repeated independently three times.

Western blot analysis. Ovarian cancer cells were lysed in RIPA buffer plus protease inhibitors (XinShengyuan). Protein concentrations of the cell lysates were determined by bicinchoninic acid kit (BCA; cat. no. P0012, Beyotime Institute of Biotechnology). The lysates were loaded 20-60 $\mu \mathrm{g}$ per lane, separated on $10 \%$ SDS-PAGE. Then the samples were transferred onto a polyvinylidene fluoride membrane (cat. no. ISEQ00010; EMD Millpore), and blocked by incubation with $5 \%$ fat-free milk in TBST buffer $(150 \mathrm{mM} \mathrm{NaCl}, 50 \mathrm{mM}$ Tris- $\mathrm{HCl}$ and $0.5 \%$ Tween-20, $\mathrm{pH}$ 7.6) at RT for $1 \mathrm{~h}$. The membranes were incubated with primary antibodies at room temperature for $1 \mathrm{~h}$ and then with horseradish peroxide (HRP) conjugated secondary antibodies (OriGene Technologies, Inc.), such as HRP conjugated goat anti-mouse IgG (cat. no. ZB-2305) and HRP conjugated goat anti-rabbit IgG (cat. 
no. ZB-2301), at RT for $1 \mathrm{~h}$ at dilution of 1:5,000. The bands were visualized using ECL reagent (cat. no. 32106; Thermo Fisher Scientific, Inc.), and calculated using ImageJ (1.42; National Institutes of Health). The antibodies used for immunoblotting were as follows (all from Abcam): E-cadherin rabbit polyclonal antibodies (Ab; cat. no. ab92486; 1:2,000), claudin rabbit polyclonal $\mathrm{Ab}$ (cat. no. ab124512; 1:3,000), vimentin rabbit polyclonal $\mathrm{Ab}$ (cat. no. ab24525; 1:3,000), MEK1 rabbit polyclonal Ab (cat. no. ab32091; 1:1,500), phosphor-MEK1/2S298 rabbit polyclonal Ab (cat. no. ab96379; 1:2,000), ERK1/2 rabbit polyclonal Ab (cat. no. ab54230; 1:2,500), phosphorMEK1/2-T202/T204 rabbit polyclonal Ab (cat. no. ab214362; 1:2,000), rabbit tubulin polyclonal Ab (cat. no. ab15568; 1:4,000) and SUMO rabbit polyclonal Ab (cat. no. ab219724; 1:1,000). The expression of tubulin was set as the control. All assay conditions were performed in triplicate.

Immunoprecipitation (IP). IP analyses were carried out as previously described (17). The cells were suspended and lysed in $50 \mathrm{mM}$ Tris- $\mathrm{HCl}$ buffer $(\mathrm{pH} 7.5)$ with $0.5 \%$ Triton $\mathrm{X}-100$ and protease inhibitors (XinShengyuan). Then, anti-MEK1 antibodies (cat. no. ab32091; Abcam) were added to induce cellular lysis and placed on a rotating stirrer at $4{ }^{\circ} \mathrm{C}$ for 2 h. Then, protein-G Sepharose 4 Fast Flow beads $(30 \mu$; Sigma-Aldrich; Merck KGaA) were added according to the manufacturer's protocol. The solutions were rotated overnight at $4^{\circ} \mathrm{C}$, and the beads were washed 3 times with Tris-HCl buffer (50 mM; pH 7.5) with $0.5 \%$ Triton X-100. In one experiment, the immune complexes were solubilized in SDS-PAGE loading buffer and separated on SDS-PAGE gels (12\%) following western blotting to detect sumo-MEK1 with anti-SUMO antibodies (cat. no. ab219724; Abcam).

Xenograft tumors of human ovarian cancer cells. The use and care of animals were approved by 'Animal Ethics Committee of Affiliated Hospital of Shandong University of Traditional Chinese Medicine' (permit no. 2018-010). All experimental procedures were carried out in accordance with the approved guidelines, and every effort was made to minimize the suffering of animals. Animals were kept in a specific pathogen-free environment, in positive pressure rooms with filtered and humidified air. The animal housing included a temperature of $23 \pm 2{ }^{\circ} \mathrm{C}$, a controlled light and dark cycle (12 h: $12 \mathrm{~h}$ ), ad libitum food and ultra-filtered water, 50-55\% humidity, ventilated caging systems and standardized environmental enrichment.

Human ovarian cells SK-OV-3 were cultured in Dulbecco's modified Eagle's medium supplemented with $10 \%$ FBS at $37^{\circ} \mathrm{C}$ with $5 \% \mathrm{CO}_{2}$. Specific pathogen-free female athymic nude mice (5-6 week old; 16-18 g; female) were purchased from Beijing HFK Bioscience and were housed three per cage under specific pathogen-free conditions. Approximately $5 \times 10^{6}$ cells were injected subcutaneously into the right flank of 5 - to 6 - week-old female athymic nude mice. The mice were divided into three groups ( $n=3$ per group) and treated with various doses of monensin ( 0,8 or $16 \mathrm{mg} / \mathrm{kg}$ body weight) at 3, 6, 9 and 12 days. Monensin was dissolved in $100 \%$ ethanol to prepare $12 \mathrm{mg} / \mathrm{ml}$ and $24 \mathrm{mg} / \mathrm{ml} \mathrm{drug} \mathrm{storage} \mathrm{solution;}$ $100 \%$ ethanol was used as the control group. Before injection, $20 \mu \mathrm{l}$ drug preservation solution or $100 \%$ ethanol was added to $\sim 180 \mu \mathrm{l}$ sterile PBS (plus 1\% penicillin and streptomycin) and diluted into $200 \mu \mathrm{l}$ working solution. Each dose was calculated according to the body weight of nude mice as follows: $8 \mathrm{mg} / \mathrm{kg}$ in the low-concentration group, $16 \mathrm{mg} / \mathrm{kg}$ in the high-concentration group, and $0 \mathrm{mg} / \mathrm{kg}$ in the control group (containing only $10 \%$ ethanol). It is necessary to mix the drug solutions before injection. The weight and palpable tumors were typically observed within 2 weeks after injection of cells, and tumor sizes were measured with a caliper 15 days after cell injection. Tumor volumes were calculated using the following formula: volume $\left(\mathrm{mm}^{3}\right)=$ length $\mathrm{x}$ width ${ }^{2} / 2$. The maximum diameter of a single subcutaneous tumor was $<1 \mathrm{~cm}$. All mice were sacrificed at 3 weeks by inhalation of $\mathrm{CO}_{2}$ gas, with a flow rate $20 \%$ replacement of home cage volume $/ \mathrm{min}$. Death was verified by cessation of the heartbeat and lack of movement, and the $\mathrm{CO}_{2}$ flow was maintained for at least 3 min after cardiac arrest. The tumors were retrieved for western blotting assay. Multiple tumors were not observed in any of the mice.

Statistical analysis. Data are expressed as the mean \pm standard deviation and analysis performed using SPSS v19.0 (IBM Corp.). All assays were performed in triplicate and/or repeated three times. Statistical significances were determined by one-way ANOVA test and Tukey's test. $\mathrm{P}<0.05$ was considered to indicate a statistically significant difference.

\section{Results}

Monensin inhibits the cell proliferation of human ovarian cancer cells. The inhibitory effects of antibiotic monensin on human ovarian cancer were initially tested on SK-OV-3 cells by CCK-8 assay. SK-OV-3 is commonly used as a model for ovarian cancer to detect the effects and potency of various chemicals. Sub-confluent SK-OV-3 cells were treated with 0 , $0.2,1$ and $5 \mu \mathrm{M}$ monensin for 24,48 or $72 \mathrm{~h}$, respectively, and then detected. CCK-8 cell viability assay suggested that cell proliferation was suppressed with increased monensin concentration and incubation duration. As low as $0.2 \mu \mathrm{M}$ monensin showed an inhibitory effect, whereas completely inhibited cell proliferation was observed in the first $24 \mathrm{~h}$ as high as $5 \mu \mathrm{M}$ monensin (Fig. 1A). CTG luminescent cell viability assay determined metabolically active cells according to the quantification of the ATP present. Based on the luminescence signal, CTG assay results indicated a similar inhibitory effect of monensin on cell proliferation with CCK-8 assay (Fig. 1B).

In order to validate the inhibitory effects of monensin on the proliferation of ovarian cancer cell SK-OV-3, monensin was applied on other ovarian cancer cell lines including A2780, OVCAR3 and CAOV-3. Cell viability was detected by CCK-8 and CTG assays. When increased concentration of monensin $(0.2,1$, and $5 \mu \mathrm{M})$ were added to the culture, all ovarian cancer cells showed similar results at $48 \mathrm{~h}$ post-treatment; their proliferative abilities were all effectively restrained by monensin (Fig. 1C and D).

Meanwhile, cell cycle distribution was analyzed by flow cytometry to detect the effect of monensin. The SK-OV-3 cells at the $\mathrm{G}_{2} / \mathrm{M}$ phase was significantly decreased from 19.17 to $12.82 \%$ when treated with $5 \mu \mathrm{M}$ monensin (Fig. $1 \mathrm{E}$ and $\mathrm{F}$ ). The A2780 cells showed similar results, and the ratio of $\mathrm{G}_{2} / \mathrm{M}$ phase was decreased from 19.64 to $13.37 \%$ when $5 \mu \mathrm{M}$ 

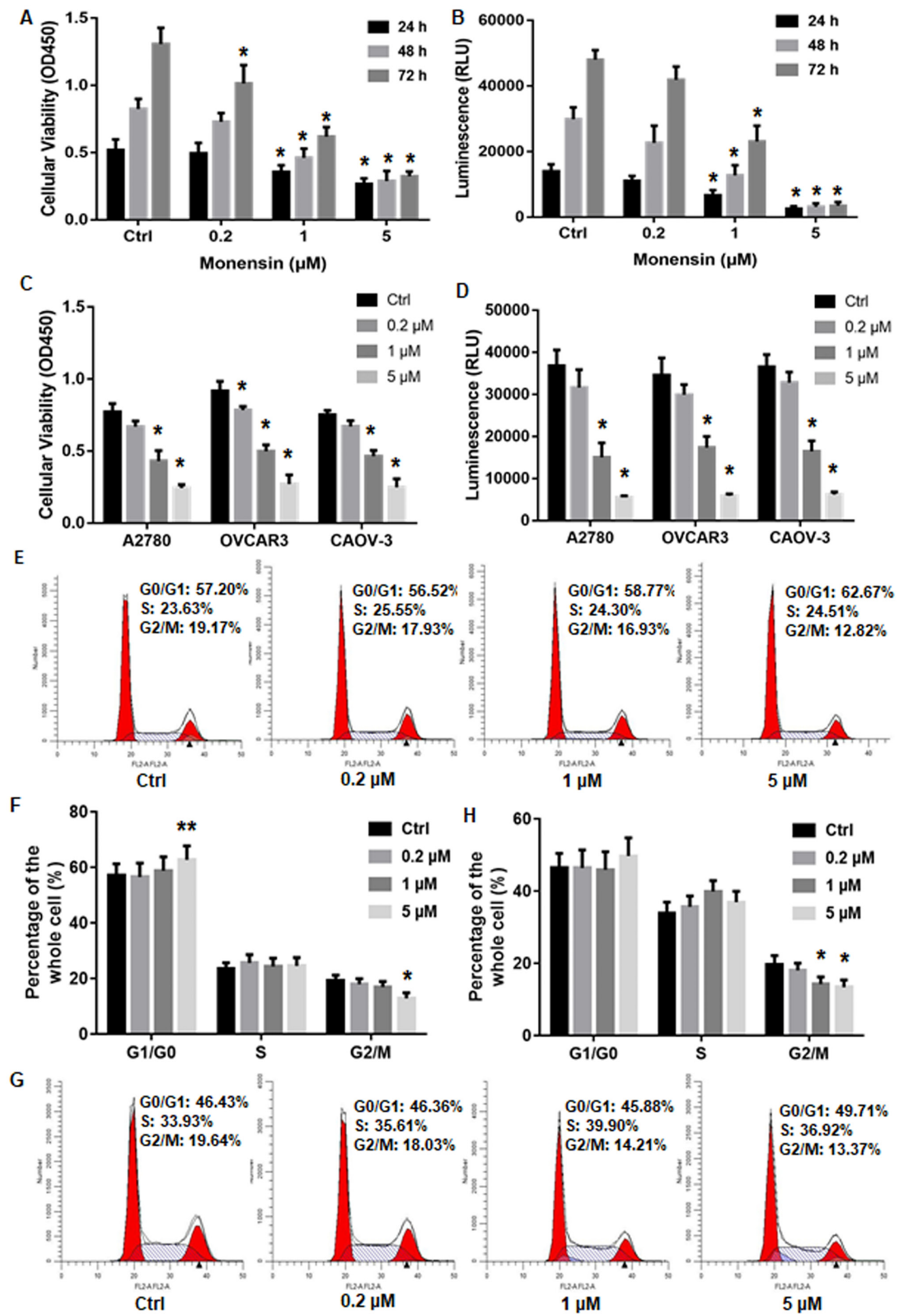

Figure 1. Monensin inhibits ovarian cancer cell proliferation. (A) CCK-8 assay was performed to detect cell viability at 24,48 and $72 \mathrm{~h}$ after treatment with different concentrations of monensin based on absorbance at $450 \mathrm{~nm}$. (B) CTG assay was used to detect cell viability at indicated time point and concentrations based on luminescence. (C and D) Cell viability of A2780, OVCAR3 and CAOV-3 were detected at $48 \mathrm{~h}$ after treatment with indicated concentrations based on CCK-8 and CTG assays. (E and F) Percentage of cells at each phase was analyzed, cells were treated with monensin for 24 h. (G and H) Cell cycle phase distributions of A2780 were determined by flow cytometry at $24 \mathrm{~h}$ after treatment with indicated concentrations of monensin. The control cells received the same concentration of the vehicle used in other groups. ${ }^{*} \mathrm{P}<0.01,{ }^{* *} \mathrm{P}<0.05$, vs. control. CCK-8, Cell Counting Kit-8; CTG, CellTiter-Glo.

monensin was applied (Fig. 1G and $\mathrm{H}$ ). The aforementioned results indicated that monensin inhibited ovarian cancer cell proliferation by blocking DNA replication.

Monensin inhibits cell invasion and malignant transformation of human ovarian cancer cells. Migration, invasion and transformation are key procedures and important features of malignant cancer. In the Transwell assay, cells treated with $0.2 \mu \mathrm{M}$ monensin had similar colon number and passingthrough-membrane percentage compared with the control group. However, the number of Transwell cells dropped to about 100 , whereas the percentage was decreased to $30 \%$ 
A

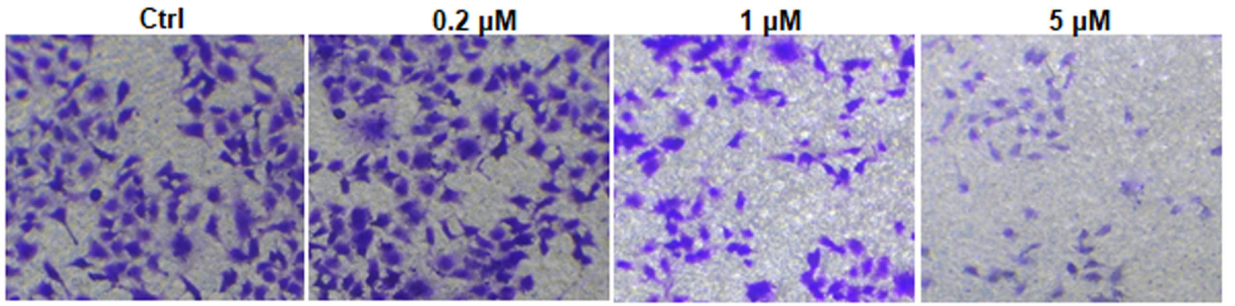

B
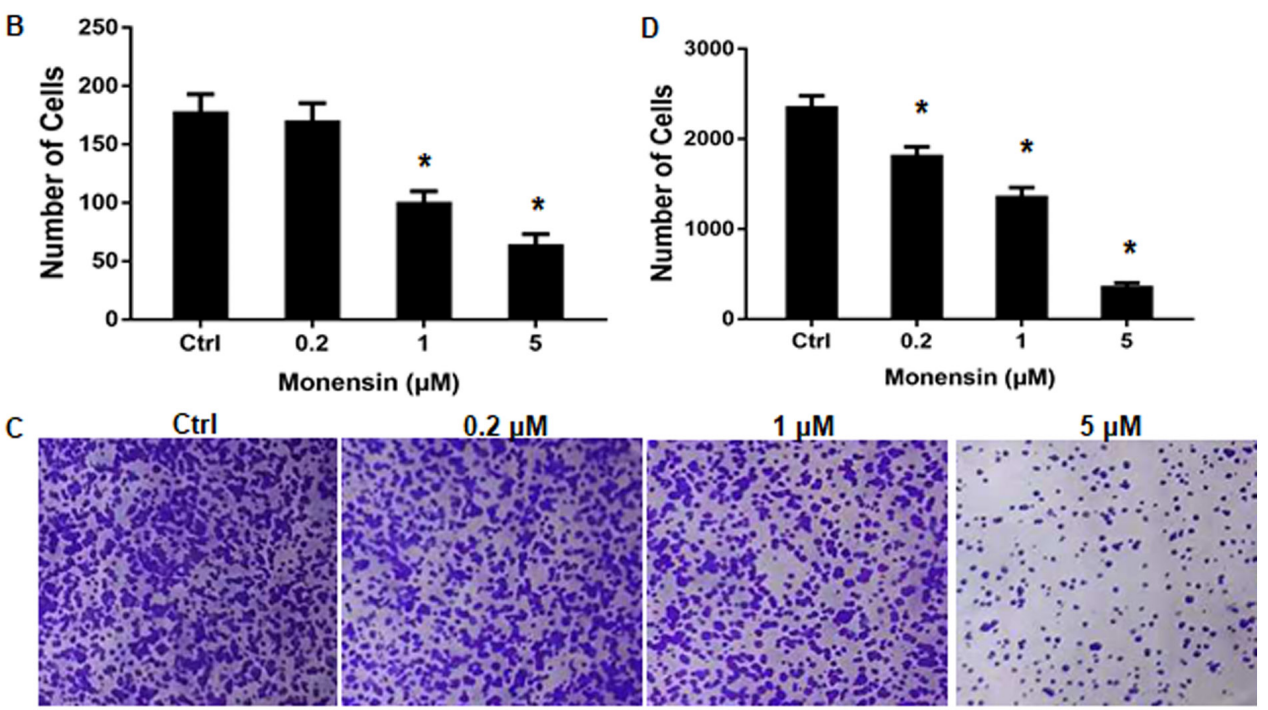

$\mathrm{E}$
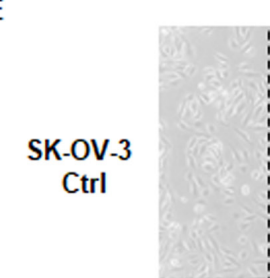

$0 \mathrm{~h}$

$24 \mathrm{~h}$
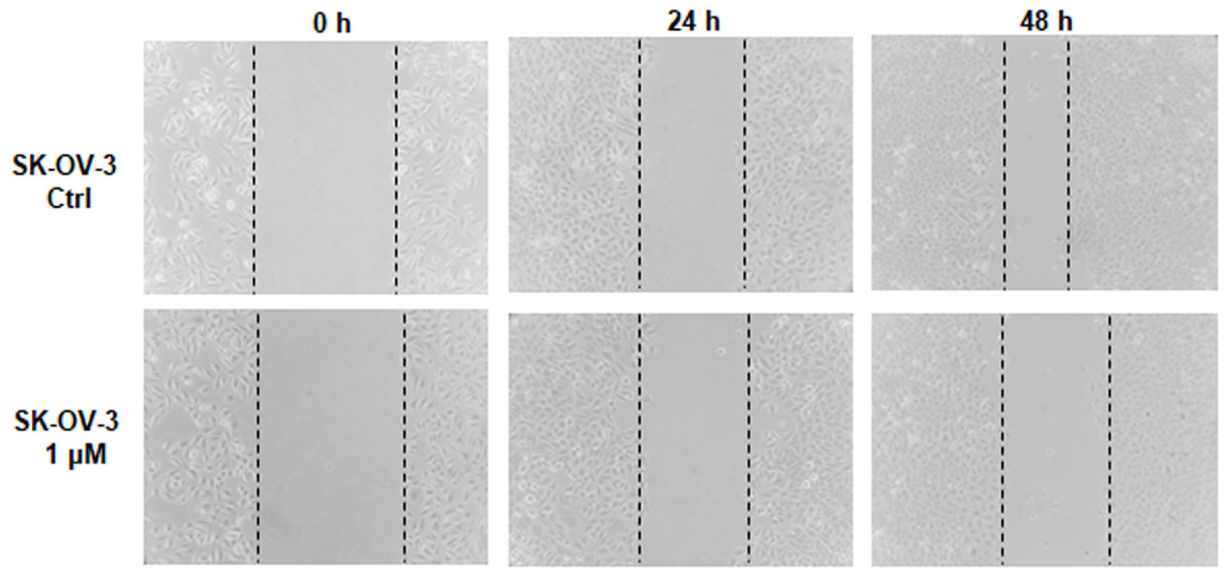

$\mathrm{F}$

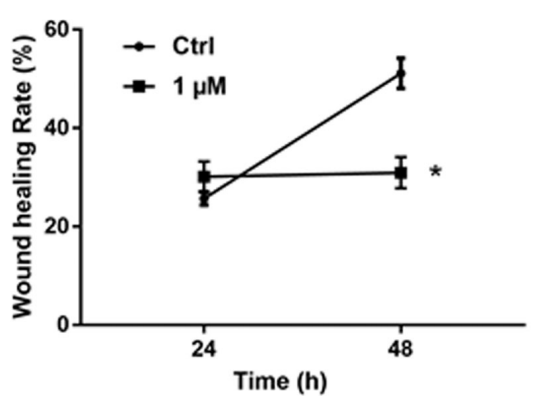

Figure 2. Monensin inhibits the invasion of ovarian cancer cells. (A) In Transwell assay, different concentrations of monensin was applied to SK-OV-3 cells, the migrating cells wells were stained and analyzed at $48 \mathrm{~h}$ after treatment. Magnification, $\mathrm{x} 400$. Cell numbers were quantified in (B). (C) In colony-formation assay, after 8-day semi-solid culture at indicated concentrations of monensin, SK-OV-3 cells were stained. Colony numbers were also analyzed in (D). (E) Wound-scratch assay was performed to assess the migration of SK-OV-3 cells; the distances were detected at 24 and $48 \mathrm{~h}$ after treatment with $1 \mu \mathrm{M}$ monensin. Magnification, x200. Wound-healing rate were analyzed in (F). The control cells received the same concentration of the vehicle used in other groups. " $\mathrm{P}<0.01$, vs. control. Ctrl, control.

when $1 \mu \mathrm{M}$ monensin was applied to cells. The number and percentage dropped dramatically to 50 and $10 \%$ when more monensin was added $(5 \mu \mathrm{M})$ (Fig. $2 \mathrm{~A}$ and B). Overall, a series of concentrations $(0,0.2,1$ and $5 \mu \mathrm{M})$ of monensin was applied in the Transwell assay, and the ability of malignant transformation of human ovarian cancer showed significant impairment and does-dependent effect (Fig. 2C and D). Moreover, when $1 \mu \mathrm{M}$ monensin was applied to cells for $48 \mathrm{~h}$, the gap closure 

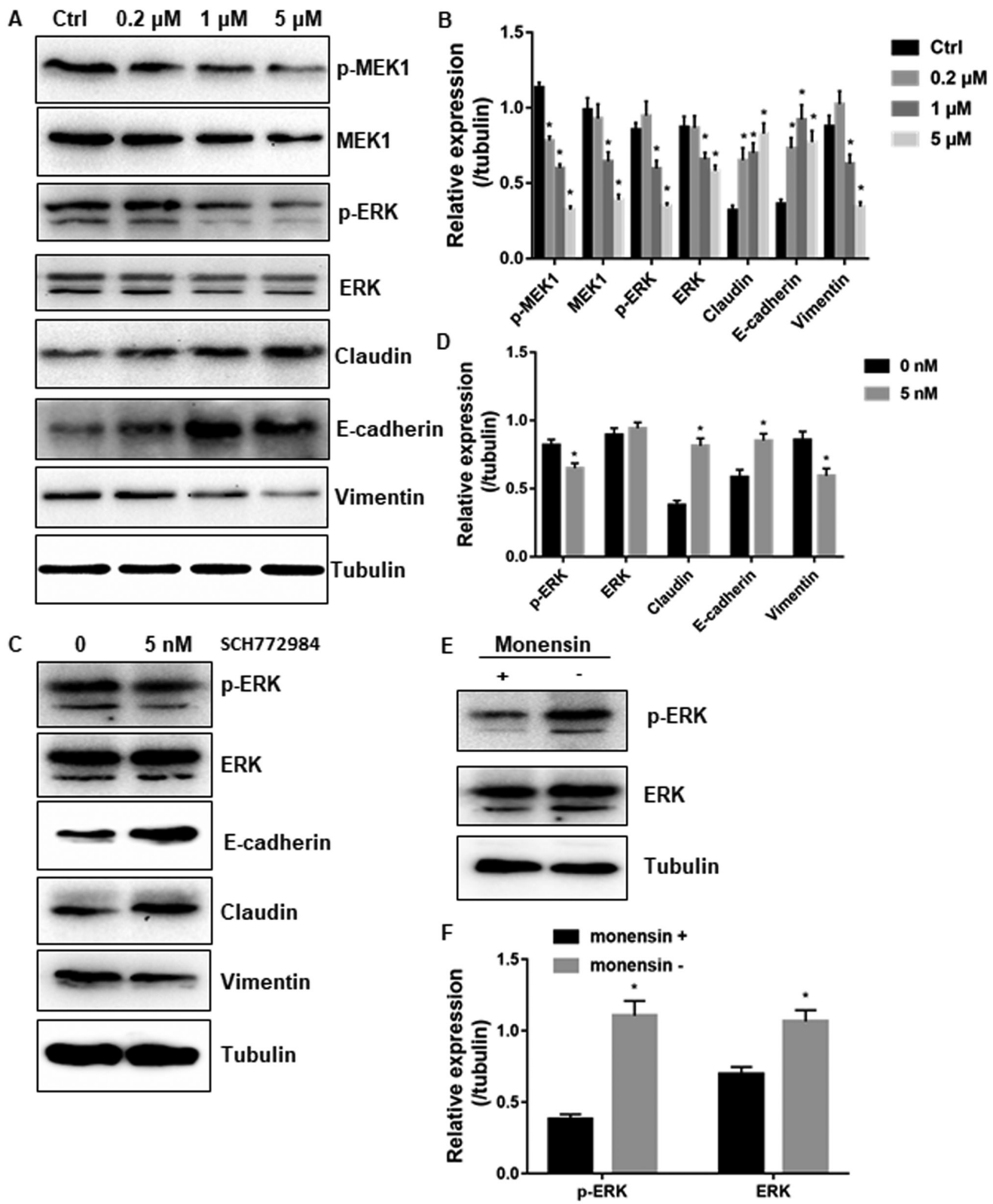

Figure 3. Monensin inhibits the MEK/ERK pathway and epithelial-mesenchymal transition. (A) p-MEK, ERK, total MEK, ERK, E-cadherin, vimentin and claudin expression levels were measured by western blotting at indicated concentrations of monensin. The densitometry analysis is shown in (B). (C) The inhibitor of ERK, SCH772984, was used to detect the E-cadherin, vimentin and claudin expression levels by western blotting, and gray scale scanning analysis is shown in (D). (E) Monensin was removed after incubation for $24 \mathrm{~h}$, and the p-ERK level in cells $12 \mathrm{~h}$ after monensin removal was detected by western blotting. The densitometry analysis is shown in (F). All assay conditions were conducted in triplicate, and the representative results are presented. ${ }^{*} \mathrm{P}<0.01$ vs. control. p-, phosphorylated.

showed time-dependent decrease (Fig. 2E), and the wound healing rate of cells treated with $1 \mu \mathrm{M}$ monensin was significantly decreased compared with the control group (Fig. 2F).

Monensin restrains the EMT-associated signaling pathway. Next, the EMT-associated signaling molecules were investigated in ovarian cancer cell lines. E-cadherin and claudin are EMT promoters, and vimentin enables dynamic cell elongation. In SK-OV-3 cells treated with monensin, the expression level of E-cadherin and claudin increased to $30 \%$ of the control, whereas the expression level of vimentin decreased to half of the control since monensin accumulated to $5 \mu \mathrm{M}$. The aforementioned findings showed a concentration-dependent response (Fig. 3A and B).
Moreover, E-cadherin and claudin can be activated by the RAS-RAF-MEK-ERK cascade, and the potential attenuation of this pathway by monensin was evaluated by western blotting. The results revealed a specific decrease in the levels of phosphorylated ERK and MEK protein in SK-OV-3 cells treated with monensin compared with the control cells. The total MEK and ERK levels were also decreased when treated with monensin. The regulation of the MEK-ERK pathway was highly associated with cell proliferation (18), which explained the monensin-induced growth inhibition. The specific inhibitor of ERK, SCH772984, served as a positive control, which showed similar results (Fig. 3C and D). Recovery assay further showed that monensin inhibited ERK phosphorylation. Monensin was removed after $24 \mathrm{~h}$ of incubation, and the 
A

IP: MEK1

Ctrl $\quad 0.2 \mu \mathrm{M} 1 \mu \mathrm{M} \quad 5 \mu \mathrm{M}$

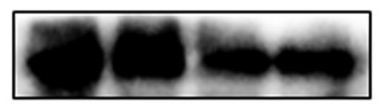

MEK1

C

IP: MEK1

IB: SUMO1
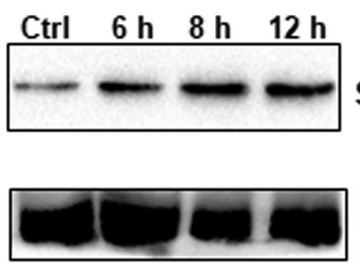

MEK1

Monensin $(1 \mu \mathrm{M})$
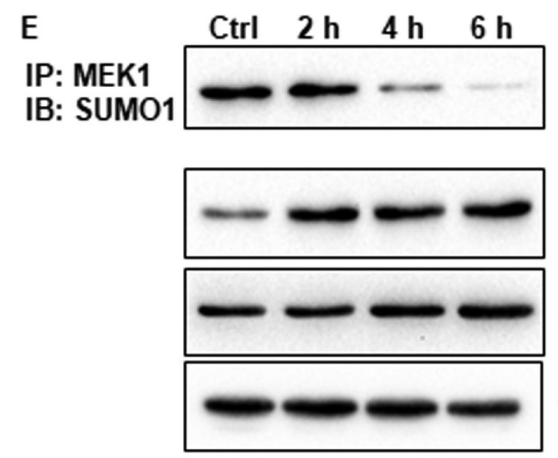

p-MEK1

MEK1

Tubulin

G

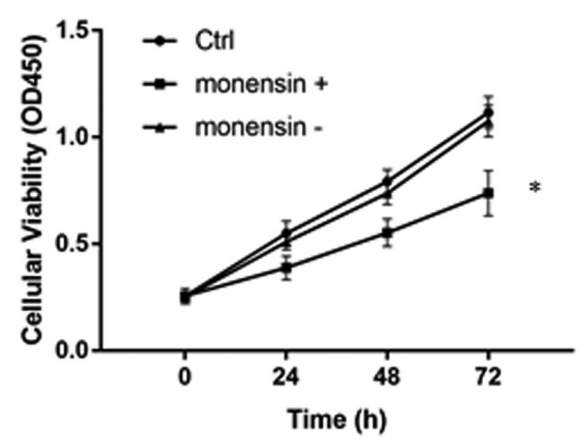

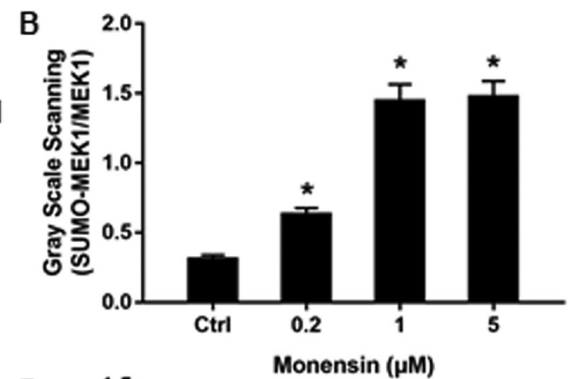

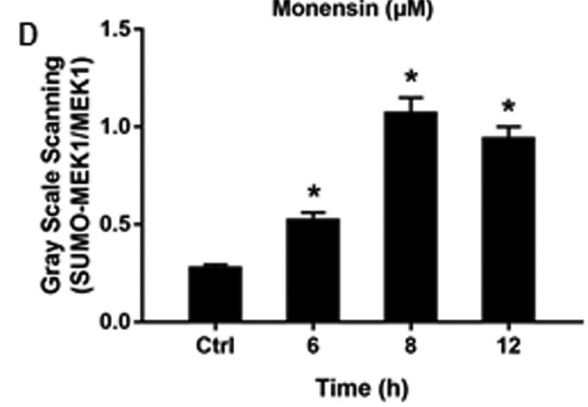

F

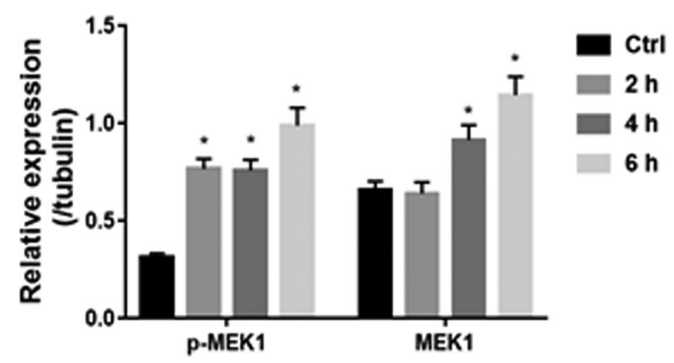

Figure 4. Monensin enhances the SUMOylation of MEK1 in ovarian cancer cells. (A) SUMOylation level of MEK1 was tested by co-IP at indicated concentrations of monensin. The gray scale scanning was used to analyze the differences between each group (B). (C and D) SUMOylation level of MEK1 was detected by co-IP with increasing treatment time. The gray scale scanning was used to analyze the differences between each group (D). (E) Cells were treated with monensin for $24 \mathrm{~h}$, then removed, SUMOylating level of MEK1 was detected at indicated time points. The densitometry analysis of p-MEK1 and MEK is shown in (F). (G) Cell viability was measured after drug withdrawal. "P<0.01 vs. control. Co-IP, co-immunoprecipitation; Ctrl, control; p-, phosphorylated; IB, immunoblot.

phospho-ERK level in cells after $12 \mathrm{~h}$ of monensin removal became much higher compared with that of cells incubated with monensin (Fig. 3E and F).

Monensin enhances the SUMOylation of MEK1. Protein SUMOylation is associated with various cellular functions, such as cell proliferation and invasion. MEK1 SUMOylation is an important way to regulate the MEK-ERK pathway. To determine whether MEK underwent SUMOylation in ovarian cancer cell line, MEK1 was expressed together with SUMO1 in HEK293 cells. MEK1 was immunoprecipitated with anti-
MEK1 monoclonal antibodies and protein G, separated by SDS-PAGE, and detected with anti-SUMO monoclonal antibodies (Fig. 4A and B). The results indicated that monensin enhanced the SUMOylation modification of MEK1 with increased drug concentration and growth of drug action time (Fig. 4C and D).

To identify the function of monensin on MEK SUMOylation and MEK-ERK phosphorylation cascade, cells transfected with MEK1 and SUMO1 were incubated with the drug for $24 \mathrm{~h}$ and the fresh medium was changed to a fresh one. At the time points of 2, 4 and $6 \mathrm{~h}$, SUMOylated MEK1, 
A

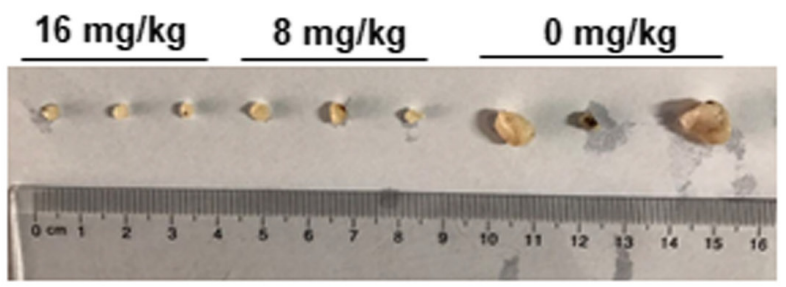

B

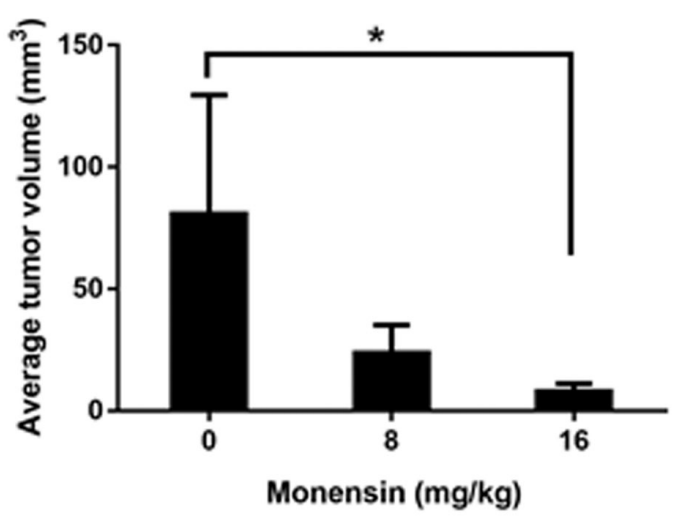

D

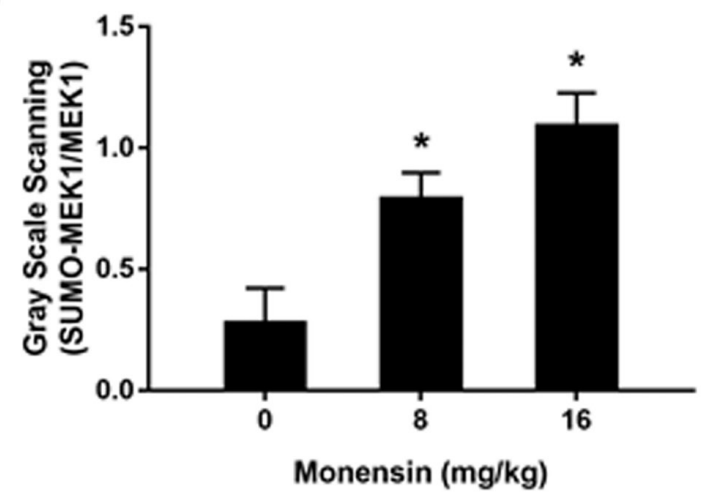

C

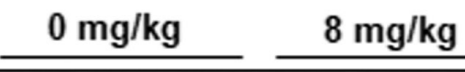
$16 \mathrm{mg} / \mathrm{kg}$

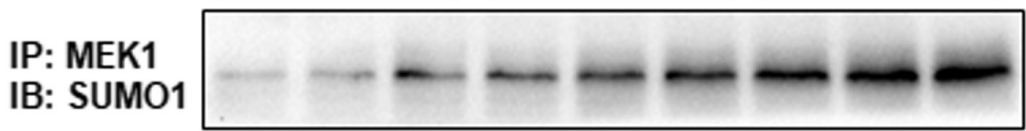

SUMO-MEK1

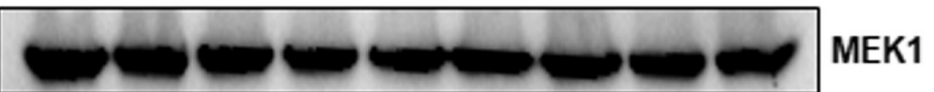

Figure 5. Monensin inhibits tumor growth and enhances MEK1 SUMOylation in the xenograft model of human ovarian cancer cells. (A) The images of tumors of the three groups. (B) Average tumor volume for each group was quantified. (C) Co-immunoprecipitation assay for SUMOylation of MEK1 in retrieved tumor samples. The gray scale scanning was used to analyze the differences between each group (D). * $\mathrm{P}<0.01$ vs. control. IP, immunoprecipitation; IB, immunoblot.

phosphorylated MEK1 and total MEK1 were detected by IP analysis. The results indicated that SUMO modification was detached upon drug removal, thereby confirming the effects of monensin on MEK1 SUMOylation. Moreover, with the withdrawal of SUMO modification, the phosphorylation of MEK1 was increased (Fig. 4E and F), whereas cell viability and proliferation were recovered (Fig. 4G). It can be concluded that SUMOylation significantly attenuated MEK1 activity in ovarian cancer cells by downregulating ERK signaling, and the process was reversible.

Monensin suppresses xenograft tumor growth and enhanced MEK1 SUMOylation in vivo. Monensin exerted significant effects on the proliferation, migration and invasion of ovarian cancer cells in vitro. Accordingly, the anticancer effects of monensin on the establishment and progression of ovarian cancer was explored in vivo. SK-OV-3 ovarian cancer cells were injected subcutaneously into the flanks of nude mice. At 3 days post-injection, the animals were treated with three doses $(0,8$ and $16 \mathrm{mg} / \mathrm{kg})$ of monensin. The tumor masses were retrieved on day 20 , and the control group had significantly larger individual tumors and higher average tumor volume compared with monensin-treated groups (Fig. 5A and B). Besides, MEK1 SUMOylation was strengthened with increased monensin dosage in vivo, consistent with the data in vitro (Figs. 5C and 4D). Together, monensin effectively inhibited tumor growth in ovarian cancer xenograft model by MEK1 SUMOylation reinforcement.

\section{Discussion}

Ovarian cancer is the most lethal tumor type among malignancies in females with high incidence and mortality rate. Most patients are diagnosed at a late stage (III and IV) and undergo different responses to chemotherapy (1). A gap currently exists between efficient treatment and clinical agents for ovarian cancer. Thus, the demand for developing effective and novel therapies to ovarian cancer is urgent.

Monensin has been approved by the Food and Drug Administration as a safety medicine in veterinary medicine. Several studies have also indicated its inhibitory effects on different types of cancer, such as prostate, melanoma and acute myeloid leukemia $(15,17,18)$. Monensin can work by freely passing through the lipid bilayer of the cytoplasmic membrane along with passive diffusion (19), but the mechanism on anticancer activity is unclear. The present study explored the possible mechanism underlying the effects of monensin on ovarian cancer.

Firstly, monensin was found to suppress the proliferation of different type of ovarian cells to minor varying degrees. Cell-cycle distribution analysis verified the inhibitory effect of monensin at the $\mathrm{G}_{1} / \mathrm{S}$ phase. Meanwhile, monensin was 
detected to inhibit the invasion and migration of ovarian cells, as well as to be highly associated with the expression of EMT-associated signaling molecules (such as E-cadherin, claudin and vimentin) and the central kinase of MEK and ERK $(20,21)$. E-cadherin is required for progression through EMT, which contributes to the loss of adherent junctions (22), whereas vimentin is an intermediate filament composition that changes cytoskeletal and polarity complex proteins and in turn contributes to EMT (23). Claudin-linked protein complexes exist between adjacent epithelial or endothelial cells, and the dissolution of tight junctions during EMT is accompanied by decreased Claudin (24). Overall, E-cadherin, claudin and vimentin were all associated with accelerated tumor growth, invasion and poor prognosis.

Furthermore, it was found that monensin intensified the SUMO1 modification of MEK1, which attenuated MEK1 activity by inhibiting MEK1 activation and negatively regulated the ERK pathway, finally suppressing cellular overgrowth. The aforementioned finding suggested that SUMO modification was a reversible and highly dynamic process, which changed with drug concentration and duration time. Results of in vivo xenograft studies further confirmed that monensin effectively inhibited xenograft tumor growth by inhibiting cell proliferation and enhancing the SUMOylation of MEK. SUMOylation is an important modulation of protein function and cell fate, and is highly responsive to various stimuli and cellular microenvironment (25).

Together, the results of the present study strongly suggested that monensin functioned as an anti-ovarian cancer agent and provided first evidence that the SUMO1 modification of MEK played crucial roles in ovarian carcinogenesis. The present study can guide future studies on the anticancer efficacy of monensin in preclinical and clinical studies. In the present study, monensin was shown to inhibit the MEK-ERK pathway and EMT, through in vitro and in vivo experiments, by enhancing MEK1 SUMOylation.

\section{Acknowledgements}

Not applicable.

\section{Funding}

This study was supported by Funds for Teachers' research of Jining Medical University (grant no. JYFC2019FKJ136).

\section{Availability of data and materials}

The datasets used and/or analyzed during the current study are available from the corresponding author on reasonable request.

\section{Authors' contributions}

SZ conceived and supervised the study; SY designed experiments and wrote the original manuscript; WW established the xenograft model; BZ and XC cultured cells and performed cell-based assay; HY analysed the data. SY and SZ confirm the authenticity of all the raw data. All authors read and approved the final manuscript.

\section{Ethics approval and consent to participate}

The use and care of animals were approved by 'Animal Ethics Committee of Affiliated Hospital of Shandong University of Traditional Chinese Medicine' (permit no. 2018-010).

\section{Patient consent for publication}

Not applicable.

\section{Competing interests}

The authors declare that they have no competing interests.

\section{References}

1. Siegel RL, Miller KD and Jemal A: Cancer statistics, 2015. CA Cancer J Clin 65: 5-29, 2015.

2. Phillips-Chavez C, Watson M, Coward J and Schloss J: A systematic literature review assessing if genetic biomarkers are predictors for platinum-based chemotherapy response in ovarian cancer patients. Eur J Clin Pharmacol 76: 1059-1074, 2020.

3. Zhang R, Shi H, Ren F, Feng W, Cao Y, Li G, Liu Z, Ji P and Zhang M: MicroRNA-338-3p suppresses ovarian cancer cells growth and metastasis: Implication of Wnt/catenin beta and MEK/ERK signaling pathways. J Exp Clin Cancer Res 38: 494, 2019.

4. Wen J, Zhao Z, Huang L, Wang L, Miao Y and Wu J: IL-8 promotes cell migration through regulating EMT by activating the Wnt $/ \beta$-catenin pathway in ovarian cancer. J Cell Mol Med 24: $1588-1598,2020$.

5. Kan T, Wang W, Ip PP, Zhou S, Wong AS, Wang X and Yang M: Single-cell EMT-related transcriptional analysis revealed intracluster heterogeneity of tumor cell clusters in epithelial ovarian cancer ascites. Oncogene 39: 4227-4240, 2020.

6. Wang S, Wang C, Li X, Hu Y, Gou R, Guo Q, Nie X, Liu J, Zhu L and Lin B: Down-regulation of TRIB3 inhibits the progression of ovarian cancer via MEK/ERK signaling pathway. Cancer Cell Int 20: 418, 2020.

7. Liu J, Hu HB, Liu YM, Li FX, Zhang LP and Liao ZM: LncRNA HOTTIP promotes the proliferation and invasion of ovarian cancer cells by activating the MEK/ERK pathway. Mol Med Rep 22: 3667-3676, 2020.

8. Wu PK, Becker A and Park JI: Growth inhibitory signaling of the Raf/MEK/ERK pathway. Int J Mol Sci 21: 21, 2020.

9. Parker MI, Nikonova AS, Sun D and Golemis EA: Proliferative signaling by ERBB proteins and RAF/MEK/ERK effectors in polycystic kidney disease. Cell Signal 67: 109497, 2020.

10. Nayak A and Amrute-Nayak M: SUMO system - a key regulator in sarcomere organization. FEBS J 287: 2176-2190, 2020.

11. Varejão N, Lascorz J, Li Y and Reverter D: Molecular mechanisms in SUMO conjugation. Biochem Soc Trans 48: 123-135, 2020.

12. Wan W, Zhang X, Huang C, Chen L, Yang X, Bao K and Peng T: Monensin inhibits glioblastoma angiogenesis via targeting multiple growth factor receptor signaling. Biochem Biophys Res Commun 530: 479-484, 2020.

13. Gu J, Huang L and Zhang Y: Monensin inhibits proliferation, migration, and promotes apoptosis of breast cancer cells via downregulating UBA2. Drug Dev Res 81: 745-753, 2020.

14. Dayekh K, Johnson-Obaseki S, Corsten M, Villeneuve PJ, Sekhon HS, Weberpals JI and Dimitroulakos J: Monensin inhibits epidermal growth factor receptor trafficking and activation: Synergistic cytotoxicity in combination with EGFR inhibitors. Mol Cancer Ther 13: 2559-2571, 2014.

15. Markowska A, Kaysiewicz J, Markowska J and Huczyński A: Doxycycline, salinomycin, monensin and ivermectin repositioned as cancer drugs. Bioorg Med Chem Lett 29: 1549-1554, 2019.

16. Rajendran V, Ilamathi HS, Dutt S, Lakshminarayana TS and Ghosh PC: Chemotherapeutic potential of monensin as an antimicrobial agent. Curr Top Med Chem 18: 1976-1986, 2018.

17. Xin H, Li J, Zhang H, Li Y, Zeng S, Wang Z, Zhang Z and Deng F: Monensin may inhibit melanoma by regulating the selection between differentiation and stemness of melanoma stem cells. PeerJ 7: e7354, 2019. 
18. Yusenko MV, Trentmann A, Andersson MK, Ghani LA, Jakobs A, Arteaga Paz MF, Mikesch JH, Peter von Kries J, Stenman G and Klempnauer KH: Monensin, a novel potent MYB inhibitor, suppresses proliferation of acute myeloid leukemia and adenoid cystic carcinoma cells. Cancer Lett 479: 61-70, 2020

19. Vanneste M, Huang Q, Li M, Moose D, Zhao L, Stamnes MA Schultz M, Wu M and Henry MD: High content screening identifies monensin as an EMT-selective cytotoxic compound. Sci Rep 9: 1200, 2019.

20. Hu M, Zhang Y, Li X, Cui P, Li J, Brännström M, Shao LR and Billig H: Alterations of endometrial epithelial-mesenchymal transition and MAPK signalling components in women with PCOS are partially modulated by metformin in vitro. Mol Hum Reprod 26: 312-326, 2020.

21. Xin L, Zhao R, Lei J, Song J, Yu L, Gao R, Ha C, Ren Y, Liu X, Liu Y, et al: SND1 acts upstream of SLUG to regulate the epithelial-mesenchymal transition (EMT) in SKOV3 cells. FASEB J 33: 3795-3806, 2019.

22. Sommariva $\mathrm{M}$ and Gagliano N: E-cadherin in pancreatic ductal adenocarcinoma: A multifaceted actor during EMT. Cells 9: 9, 2020.
23. Avtanski D, Garcia A, Caraballo B, Thangeswaran P, Marin S, Bianco J, Lavi A and Poretsky L: Resistin induces breast cancer cells epithelial to mesenchymal transition (EMT) and stemness through both adenylyl cyclase-associated protein 1 (CAP1)dependent and CAP1-independent mechanisms. Cytokine 120: 155-164, 2019.

24. Wang K, Li T, Xu C, Ding Y, Li W and Ding L: Claudin-7 downregulation induces metastasis and invasion in colorectal cancer via the promotion of epithelial-mesenchymal transition. Biochem Biophys Res Commun 508: 797-804, 2019.

25. Jansen NS and Vertegaal ACO: A chain of events: Regulating target proteins by SUMO polymers. Trends Biochem Sci 46: $113-123,2021$.

(7)(9) This work is licensed under a Creative Commons

EY NO NO Attribution-NonCommercial-NoDerivatives 4.0 International (CC BY-NC-ND 4.0) License. 